\title{
Lattice Rules and Randomized Quasi-Monte Carlo
}

\author{
Pierre L'Ecuyer \\ Département d'Informatique et de Recherche Opérationnelle \\ Université de Montréal \\ C.P.6128, Succ. Centre-Ville \\ Montréal, Québec H3C 3J7, Canada \\ Phone: +1-(514)-343-2143 \\ FAX: +1-(514)-343-5834 \\ lecuyer@iro.umontreal.ca
}

\begin{abstract}
High-dimensional multivariate integration is a difficult problem for which the Monte Carlo method is often the only viable approach. This method provides an unbiased estimator of the integral, together with a probabilistic error estimate (e.g., in the form of a confidence interval). The aim of randomized quasi-Monte Carlo (QMC) methods is to provide lower-variance unbiased estimators, also with error estimates. This talk will concentrate on one class of randomized QMC methods: randomized lattice rules.

We will explain how these methods fit into QMC methods in general and why they are interesting, how to choose their parameters, and how they can be used for medium and large-scale simulations. Numerical examples will be given to illustrate their effectiveness.
\end{abstract}

